\title{
Contraction versus Relaxation: A Comparison of Two Approaches for the Negative Cost Cycle Detection Problem
}

\author{
K. Subramani and L. Kovalchick \\ LCSEE, \\ West Virginia University, \\ Morgantown, WV \\ $\{$ ksmani, lynn $\} @ c s e e \cdot$ wvu. edu
}

\begin{abstract}
In this paper, we develop a greedy algorithm for the negative cost cycle detection problem and empirically contrast its performance with the "standard" Bellman-Ford (BF) algorithm for the same problem. Our experiments indicate that the greedy approach is superior to the dynamic programming approach of $\mathrm{BF}$, on a wide variety of inputs.
\end{abstract}

\section{Introduction}

In this paper, we are concerned with the Negative Cost Cycle Detection problem (NEG): Given a directed graph $\mathbf{G}=<\mathbf{V}, \mathbf{E}>$, where $|\mathbf{V}|=n$ and $|\mathbf{E}|=m$, and a cost function $c: \mathbf{E} \rightarrow \Re$, is there a negative cost cycle in $\mathbf{G}$ ?

Our main contribution is the proposal of a greedy algorithm for NEG, based on vertex contraction. All approaches to the negative cost cycle problem in the literature are based on dynamic programming; our approach is the first and only greedy approach to this problem, that we know of. Scaling approaches have also been proposed for NEG ([Gol95]); however, these algorithms are efficient, only when the edge-weights are small integers. We do not place any restrictions on the edge costs. We note that the problem, as specified, is a decision problem, in that all that is asked of an algorithm is to detect the presence of a negative cycle. This problem finds application in a wide variety of areas such as Constraint Analysis [DMP91], Compiler Construction [Pug92], VLSI Design [WE94] and Scheduling [Sub02].

Our experiments indicate that Vertex Contraction is an effective alternative to the "standard" Bellman-Ford (BF) algorithm for the same problem; this is most surprising since in the case of sparse graphs, BF is provably superior to Vertex Contraction (from the perspective of asymptotic analysis).

\section{The Vertex-Contraction Algorithm}

The vertex contraction procedure consists of eliminating a vertex from the input graph, by merging all its incoming and outgoing edges. Consider a vertex $v_{i}$ 
with incoming edge $e_{k i}$ and outgoing edge $e_{i j}$. When $v_{i}$ is contracted, $e_{k i}$ and $e_{i j}$ are deleted and a single edge $e_{k j}^{\prime}$ is added with cost $c_{k i}+c_{i j}$. This process is repeated for each pair of incoming and outgoing edges. Consider the edge $e_{k j}^{\prime}$ that is created by the contraction; it falls into one of the following categories:

1. It is the first edge between vertex $v_{k}$ and $v_{j}$. In this case, nothing more is to be done.

2. An edge $e_{k j}$ already existed between $v_{k}$ and $v_{j}$, prior to the contraction of $v_{i}$. In this case, if $c_{k j}^{\prime}<c_{k j}$, keep the new edge and delete the previously existing edge (since it is redundant); otherwise delete the new edge (since it is redundant).

Algorithm (2.1) is a formal description of our technique.

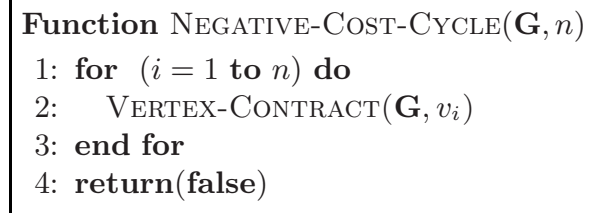

Algorithm 2.1: Negative cost cycle detection

We defer a formal proof of the correctness of the $\mathrm{VC}$ algorithm to the journal version of this paper. In the full version, an analysis of $\mathrm{VC}$ is also provided; we show that the algorithm runs in worst case time $O\left(n^{3}\right)$.

Thus, for dense graphs, Algorithm (2.1) is competitive with Bellman-Ford (BF); however for sparse graphs, the situation is not so sanguine. For instance, an adversary could provide the graph in Figure (1) as input.

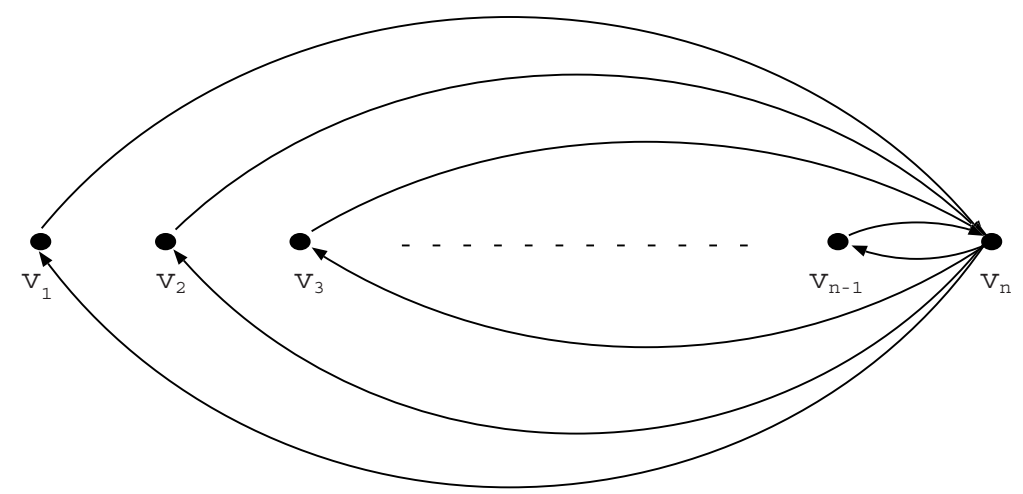

Fig. 1. Sparse graph that becomes dense after vertex contraction 


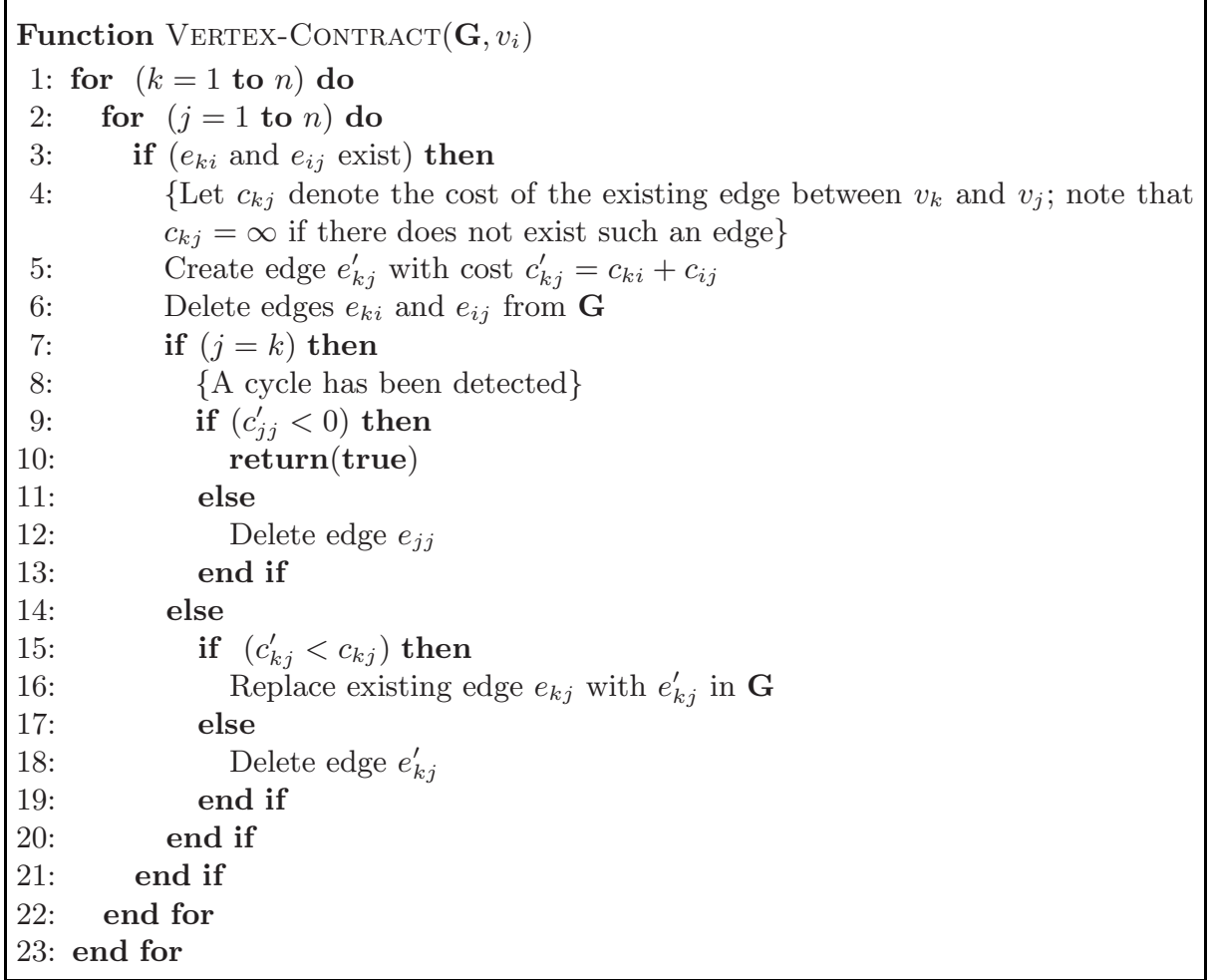

Algorithm 2.2: Vertex Contraction

The above graph is sparse and has exactly $2 \cdot(n-1)$ edges. Observe that if vertex $v_{n}$ is contracted first, the resultant graph is the complete graph on $n-1$ vertices and therefore dense. We call this graph the cruel adversary; in our experiments, we made it a point to contrast the performance of the vertex contraction algorithm with $\mathrm{BF}$ on this input. It is clear that any well-defined order of selecting the next vertex to be contracted is susceptible to attack by a malicious adversary; we could however choose the vertex to be contracted at random, without affecting the correctness of the algorithm. We have implemented Algorithm (2.1) in two different ways; in one implementation, the vertex to be contracted is chosen in a well-defined order, whereas in the second implementation, it is chosen at random. Algorithm (2.3) is a formal description of the random vertex contraction algorithm.

\section{Implementation}

Our experiments are classified into various categories, based on the following criteria:

1. Type of input graph - Sparse with many small negative cycles (Type A), Sparse with a few long negative cycles (Type B), Dense with many small 


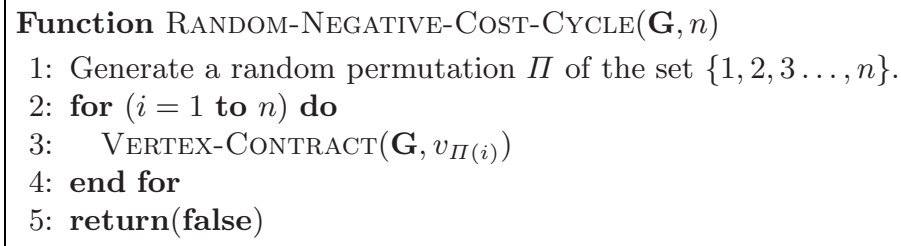

Algorithm 2.3: Random negative cost cycle detection algorithm

negative cycles (Type C), Dense with a few long negative cycles (Type D), and the Cruel Adversary (Type E).

2. Type of Algorithm - Bellman-Ford (BF), Vertex-Contraction (VC) or Random Vertex-Contraction (RVC).

3. Type of Graph Data Structure - Simple Pointer or Array of Pointers.

All times recorded were averaged over 5 executions of each implementation.

\subsection{Machine Characteristics}

Table 1. Implementation System.

\begin{tabular}{|c|c|}
\hline Machine Model & Silicon Graphics Onyx2 \\
\hline Processors & IR2/R10 250 Mhz \\
\hline Cache & $8 \mathrm{MB}$ \\
\hline Memory & $2 \mathrm{~GB}$ \\
\hline Operating System & IRIX 6.5 .15 \\
\hline Language & $\mathrm{C}$ \\
\hline Software & $\mathrm{gcc}$ \\
\hline
\end{tabular}

\subsection{Graph Data Structures}

Two different types of graph data structures were used for the experiments. We implemented BF, VC and RVC with an array of pointers structure and a simple pointer structure.

The array of pointers structure is a new representation. This representation makes use of an array of $n$ pointers, one for each of the $n$ vertices of the graph. Each pointer points to an $n$ element array, which corresponds to the $n$ vertices of the graph. Initially all entries of the array are assigned an undefined value. For a vertex $v_{i}$, if there exists an edge from $v_{i}$ to another vertex $v_{j}$, position $v_{j}$ of 
the array that $v_{i}$ points to is assigned the cost of the edge between $v_{i}$ and $v_{j}$. It should be noted that this representation is different from the adjacency-matrix representation [CLR92].

The simple pointer structure, also known as the adjacency-list representation [CLR92], requires only linear space. This representation makes use of an array of $n$ lists, one for each of the $n$ vertices of the graph.

\subsection{Experimental Setup for Sparse Graphs}

Sparse graphs were generated using the generator developed by Andrew Goldberg [CG96], which generates multiple edges between two vertices. Sparse graphs are defined as graphs with $o(n \cdot \log n)$ edges. We generated each graph 5 times using 5 different seeds for the random number generator.

Graphs of Type A and B were tested, with a number of vertices ranging from 500 to 5,500 in increments of 500 .

We define a small negative cycle as one consisting of at most $\frac{n}{100}$ vertices. We define a long negative cycle as one consisting of $\Omega\left(\frac{n}{2}\right)$ vertices. The number of long negative cycles in the input graphs was set to 4 .

\begin{tabular}{|r|c|c|}
\hline \multirow{2}{*}{$n$} & \multicolumn{2}{|c|}{$\begin{array}{c}\text { Array of } \\
\text { (Time in Seinters }\end{array}$} \\
\cline { 2 - 3 } VConds) \\
\hline 500 & 0.15351 & BF \\
750 & 0.50453 & 9.80657 \\
1,000 & 1.58202 & 27.9044 \\
1,250 & 2.23023 & 54.0744 \\
1,500 & 4.74535 & 105.143 \\
1,750 & 5.55235 & 156.953 \\
2,000 & 12.7588 & 257.852 \\
2,250 & 19.5588 & 337.136 \\
2,500 & 13.9183 & 514.046 \\
2,750 & 24.3229 & 624.652 \\
3,000 & 30.4645 & 883.024 \\
3,250 & 34.8372 & 1034.04 \\
3,500 & 49.6497 & 1400.41 \\
3,750 & 48.4852 & 1606.85 \\
4,000 & 88.8478 & 2104.50 \\
4,250 & 70.4305 & 2319.88 \\
4,500 & 132.506 & 3094.30 \\
4,750 & 82.0854 & 3180.42 \\
5,000 & 108.178 & 4229.53 \\
5,250 & 116.699 & 4377.80 \\
5,500 & 133.606 & 5453.65 \\
\hline
\end{tabular}

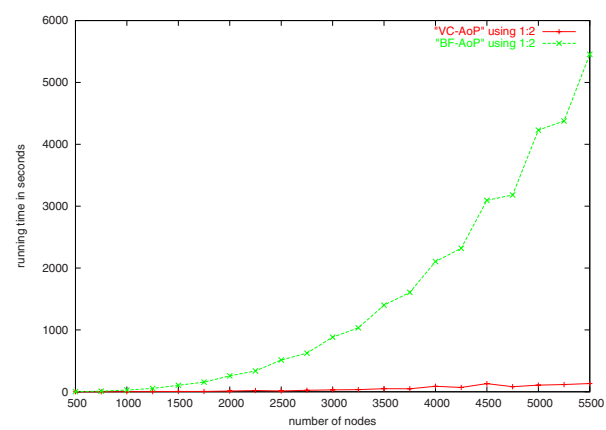

Fig. 2. Comparison of Vertex Contraction (VC), and Bellman-Ford (BF) Array of Pointer (AoP) implementation execution times (seconds) required to solve the Negative Cost Cycle problem for Type A graphs. 


\begin{tabular}{|r|c|c|}
\hline \multirow{2}{*}{$n$} & \multicolumn{2}{|c|}{ Simple Pointer } \\
\cline { 2 - 3 } (Time in Seconds) \\
\cline { 2 - 3 } VC & BF \\
\hline 500 & 0.003933 & 1.65399 \\
750 & 0.007623 & 5.19749 \\
1,000 & 0.009573 & 11.8637 \\
1,250 & 0.023780 & 22.5836 \\
1,500 & 0.013797 & 38.9001 \\
1,750 & 0.013525 & 64.8949 \\
2,000 & 0.022071 & 103.797 \\
2,250 & 0.022178 & 155.955 \\
2,500 & 0.025375 & 222.823 \\
2,750 & 0.030861 & 304.137 \\
3,000 & 0.040336 & 403.182 \\
3,250 & 0.046731 & 519.489 \\
3,500 & 0.047264 & 656.995 \\
3,750 & 0.071233 & 814.206 \\
4,000 & 0.063790 & 995.579 \\
4,250 & 0.073681 & 1199.38 \\
4,500 & 0.101693 & 1433.95 \\
4,750 & 0.083590 & 1688.46 \\
5,000 & 0.124874 & 1981.08 \\
5,250 & 0.084357 & 2295.98 \\
5,500 & 0.087477 & 2650.91 \\
\hline
\end{tabular}

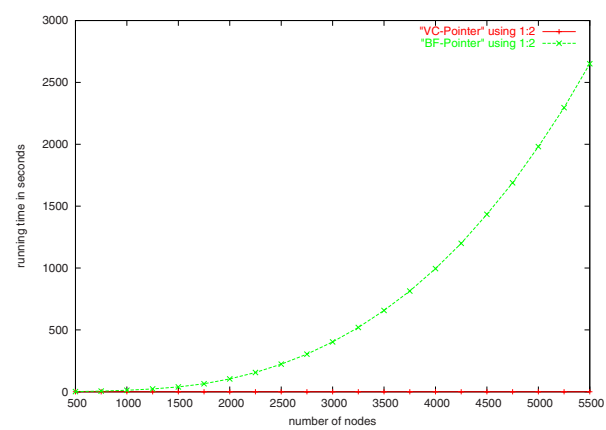

Fig. 3. Comparison of Vertex Contraction (VC), and Bellman-Ford (BF) Simple Pointer implementation execution times (seconds) required to solve the Negative Cost Cycle problem for Type A graphs.

\begin{tabular}{|c|c|c|}
\hline & \multicolumn{2}{|c|}{ Array of Pointers } \\
$n$ & (Time in Seconds) \\
\cline { 2 - 3 } & VC & BF \\
\hline 500 & 0.22578 & 2.80053 \\
750 & 0.51732 & 9.40496 \\
1,000 & 1.63598 & 25.2701 \\
1,250 & 2.76916 & 52.7881 \\
1,500 & 4.03085 & 103.765 \\
1,750 & 4.58197 & 152.435 \\
2,000 & 11.8345 & 253.484 \\
2,250 & 20.4233 & 330.726 \\
2,500 & 13.9014 & 502.027 \\
2,750 & 23.9882 & 607.284 \\
3,000 & 27.1102 & 875.921 \\
3,250 & 35.6303 & 995.875 \\
3,500 & 49.7201 & 1383.19 \\
3,750 & 48.6051 & 1577.46 \\
4,000 & 77.5183 & 2071.36 \\
4,250 & 65.2763 & 2307.49 \\
4,500 & 120.153 & 2978.97 \\
4,750 & 83.6087 & 3209.21 \\
5,000 & 92.0987 & 4076.75 \\
5,250 & 151.066 & 4376.84 \\
5,500 & 130.703 & 5408.41 \\
\hline
\end{tabular}

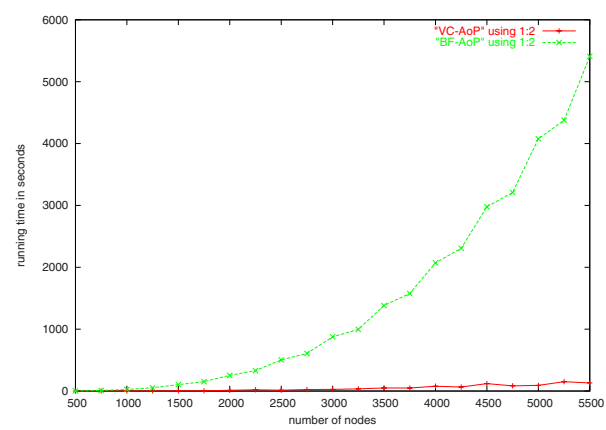

Fig. 4. Comparison of Vertex Contraction (VC), and Bellman-Ford (BF) Array of Pointer (AoP) implementation execution times (seconds) required to solve the Negative Cost Cycle problem for Type B graphs. 


\begin{tabular}{|r|c|c|}
\hline \multirow{2}{*}{$n$} & \multicolumn{2}{|c|}{ Simple Pointer } \\
\cline { 2 - 3 } (Time in Seconds) \\
\cline { 2 - 3 } VC & BF \\
\hline 500 & 0.004119 & 1.65394 \\
750 & 0.008052 & 5.20284 \\
1,000 & 0.011301 & 11.8367 \\
1,250 & 0.022105 & 22.5755 \\
1,500 & 0.099097 & 38.8326 \\
1,750 & 0.022232 & 64.7921 \\
2,000 & 0.021255 & 103.191 \\
2,250 & 0.037886 & 154.749 \\
2,500 & 0.026206 & 222.234 \\
2,750 & 0.030613 & 303.657 \\
3,000 & 0.037332 & 403.146 \\
3,250 & 0.050565 & 518.724 \\
3,500 & 0.047130 & 655.168 \\
3,750 & 0.078139 & 813.916 \\
4,000 & 0.188993 & 993.696 \\
4,250 & 0.078959 & 1199.66 \\
4,500 & 0.106838 & 1432.46 \\
4,750 & 0.059203 & 1690.80 \\
5,000 & 0.128170 & 1977.29 \\
5,250 & 0.096562 & 2293.64 \\
5,500 & 0.114865 & 2646.47 \\
\hline
\end{tabular}

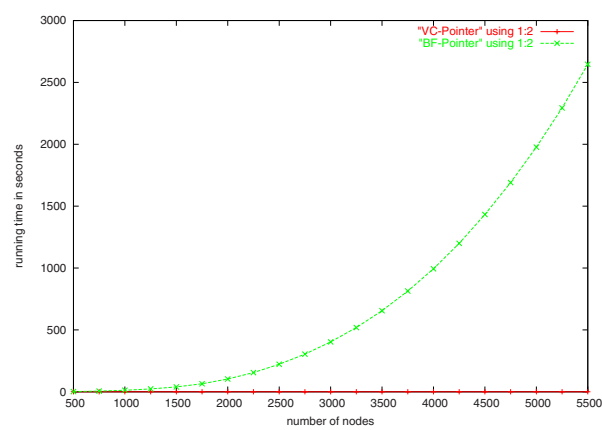

Fig. 5. Comparison of Vertex Contraction (VC), and Bellman-Ford (BF) Simple Pointer implementation execution times (seconds) required to solve the Negative Cost Cycle problem for Type B graphs.

\subsection{Conclusions}

It is easy to see from the tables and graphs in Figure (2) through Figure (5) that VC outperforms BF using either data structure; this is true for both types of sparse graphs that were tested. We conclude that $\mathrm{VC}$ is far superior to $\mathrm{BF}$ for sparse graphs.

An asymptotic analysis would indicate that BF is superior to $V C$ for dense graphs, although, our experiments contradict this indication.

\subsection{Experimental Setup for Dense Graphs}

Dense graphs were generated using the generator developed by Andrew Goldberg [CG96]. Dense graphs were defined as those with $\Omega\left(\frac{n^{2}}{8}\right)$ edges. We generated each graph 5 times using 5 different seeds for the random number generator.

Graphs of Type C and D were tested, with a number of vertices ranging from 125 to 1,875 in increments of 125 , with small negative cycles and long negative cycles defined as in Section $§ 3.3$. 


\begin{tabular}{|r|c|c|}
\hline \multirow{2}{*}{$n$} & \multicolumn{2}{|c|}{$\begin{array}{c}\text { Array of Pointers } \\
\text { (Time in Seconds) }\end{array}$} \\
\cline { 2 - 3 } & VC & BF \\
\hline 125 & 0.03830 & 0.07012 \\
250 & 0.05997 & 0.52512 \\
375 & 0.15247 & 1.74020 \\
500 & 0.28095 & 4.08695 \\
625 & 0.46288 & 7.98574 \\
750 & 0.65885 & 13.7706 \\
875 & 1.48780 & 21.9978 \\
1,000 & 1.55311 & 34.5631 \\
1,125 & 2.97252 & 51.5897 \\
1,250 & 3.37425 & 74.6079 \\
1,375 & 4.37236 & 100.182 \\
1,500 & 7.30295 & 132.641 \\
1,625 & 6.22661 & 168.864 \\
1,750 & 8.63348 & 210.632 \\
1,875 & 8.00939 & 260.838 \\
\hline
\end{tabular}

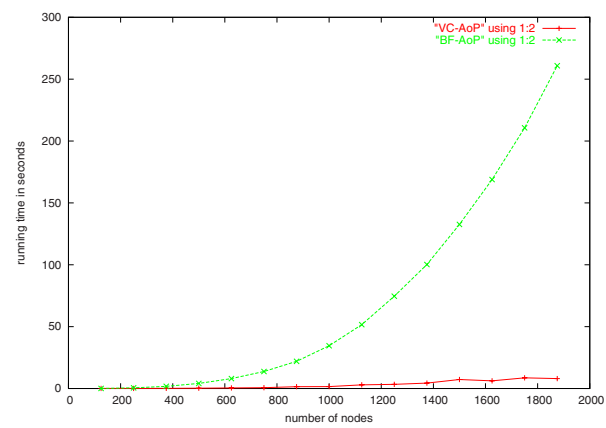

Fig. 6. Comparison of Vertex Contraction (VC), and Bellman-Ford (BF) Array of Pointer (AoP) implementation execution times (seconds) required to solve the Negative Cost Cycle problem for Type $\mathrm{C}$ graphs.

\begin{tabular}{|r|c|c|}
\hline \multirow{2}{*}{$n$} & \multicolumn{2}{|c|}{$\begin{array}{c}\text { Simple Pointer } \\
\text { (Time in Seconds) }\end{array}$} \\
\cline { 2 - 3 } & VC & BF \\
\hline 125 & 0.00048 & 0.09019 \\
250 & 0.00194 & 1.02020 \\
375 & 0.00303 & 4.50625 \\
500 & 0.00675 & 13.2450 \\
625 & 0.00750 & 30.9083 \\
750 & 0.01562 & 62.0953 \\
875 & 0.03498 & 123.824 \\
1,000 & 0.09200 & 293.591 \\
1,125 & 0.11334 & 672.256 \\
1,250 & 0.19100 & 1350.14 \\
1,375 & 0.25657 & 2447.76 \\
1,500 & 0.42457 & 4079.35 \\
1,625 & 0.41033 & 6346.27 \\
1,750 & 0.65944 & 9445.69 \\
1,875 & 0.99798 & 13480.9 \\
\hline
\end{tabular}

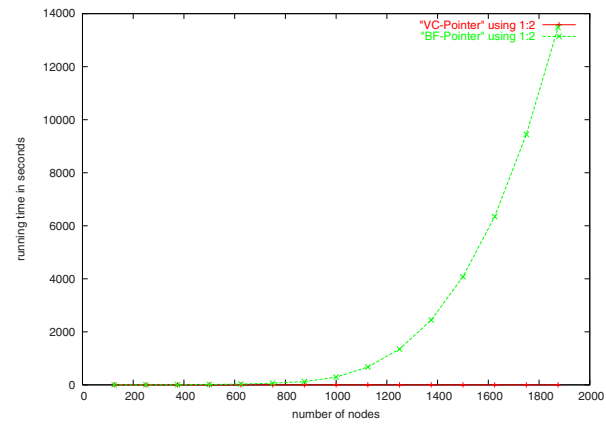

Fig. 7. Comparison of Vertex Contraction (VC), and Bellman-Ford (BF) Simple Pointer implementation execution times (seconds) required to solve the Negative Cost Cycle problem for Type C graphs. 


\begin{tabular}{|c|c|c|}
\hline \multirow{2}{*}{$n$} & \multicolumn{2}{|c|}{$\begin{array}{c}\text { Array of Pointers } \\
\text { (Time in Seconds) }\end{array}$} \\
\cline { 2 - 3 }$n$ & VC & BF \\
\hline 125 & 0.00146 & 0.06872 \\
250 & 0.06294 & 0.52747 \\
375 & 0.21069 & 1.73264 \\
500 & 0.30008 & 4.09149 \\
625 & 0.65476 & 7.98092 \\
750 & 0.74009 & 13.7807 \\
875 & 2.03858 & 21.9476 \\
1,000 & 1.50686 & 35.0864 \\
1,125 & 3.64647 & 51.5824 \\
1,250 & 3.17255 & 72.8195 \\
1,375 & 6.92824 & 101.460 \\
1,500 & 6.88772 & 133.293 \\
1,625 & 6.67336 & 167.244 \\
1,750 & 7.48221 & 212.698 \\
1,875 & 15.9974 & 263.763 \\
\hline
\end{tabular}

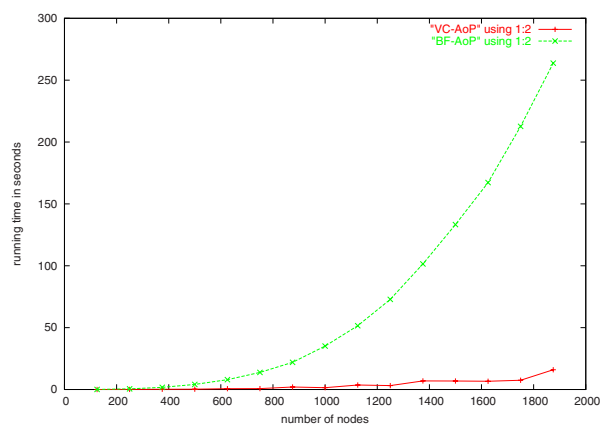

Fig. 8. Comparison of Vertex Contraction (VC), and Bellman-Ford (BF) Array of Pointer (AoP) implementation execution times (seconds) required to solve the Negative Cost Cycle problem for Type D graphs.

\begin{tabular}{|c|c|c|}
\hline \multirow{2}{*}{$n$} & \multicolumn{2}{|c|}{ Simple Pointer } \\
(Time in Seconds) \\
\cline { 2 - 3 } VC & BF \\
\hline 125 & 0.00069 & 0.08752 \\
250 & 0.00185 & 1.02023 \\
375 & 0.00488 & 4.44321 \\
500 & 0.00706 & 13.2395 \\
625 & 0.01379 & 30.6152 \\
750 & 0.01765 & 62.0956 \\
875 & 0.05033 & 122.713 \\
1,000 & 0.07456 & 293.617 \\
1,125 & 0.20491 & 664.932 \\
1,250 & 0.22301 & 1348.96 \\
1,375 & 0.41339 & 2426.95 \\
1,500 & 0.39452 & 4079.71 \\
1,625 & 1.00850 & 6299.06 \\
1,750 & 0.59050 & 9447.84 \\
1,875 & 1.43688 & 13418.7 \\
\hline
\end{tabular}

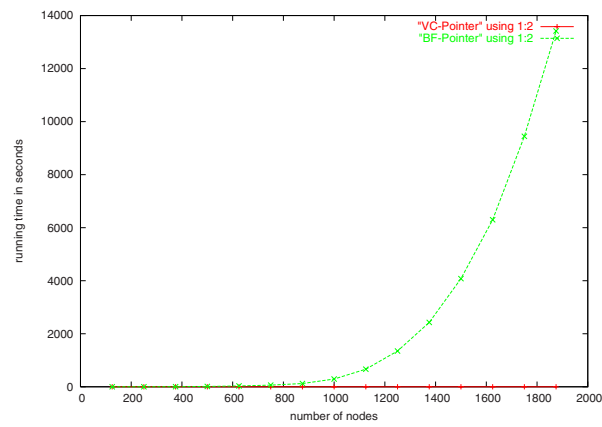

Fig. 9. Comparison of Vertex Contraction (VC), and Bellman-Ford (BF) Simple Pointer implementation execution times (seconds) required to solve the Negative Cost Cycle problem for Type D graphs.

\subsection{Conclusions}

It is easy to see from the tables and graphs in Figure (6) through Figure (9) that $\mathrm{VC}$ outperforms BF using either data structure; this is true with both types of dense graphs that were tested. We conclude that $\mathrm{VC}$ is far superior to $\mathrm{BF}$ for dense graphs. 


\subsection{Experimental Setup for Cruel Adversary Graphs}

The cruel adversary is generated by specifying the number of vertices in the graph and the maximum cost for any edge.

For our experiments we generated graphs with vertices ranging from 125 to 1,875 in increments of 125 .

\begin{tabular}{|r|c|c|c|}
\hline \multirow{2}{*}{$n$} & \multicolumn{4}{|c|}{$\begin{array}{c}\text { Array of Pointers } \\
\text { (Time in Seconds) }\end{array}$} \\
\cline { 2 - 4 } & VC & RVC & BF \\
\hline 125 & 0.02168 & 0.05125 & 0.04738 \\
250 & 0.16735 & 0.40114 & 0.34556 \\
375 & 0.55377 & 1.35226 & 1.13898 \\
500 & 1.29808 & 3.08334 & 2.66123 \\
625 & 2.54713 & 6.22039 & 5.19088 \\
750 & 4.38616 & 10.7309 & 9.02499 \\
875 & 7.01832 & 15.6965 & 14.3931 \\
1,000 & 10.9170 & 25.7463 & 23.6483 \\
1,125 & 15.7832 & 37.1957 & 35.7989 \\
1,250 & 21.9961 & 52.2092 & 54.5719 \\
1,375 & 30.1900 & 71.4197 & 72.7322 \\
1,500 & 41.3305 & 93.0058 & 94.8282 \\
1,625 & 53.3580 & 66.8170 & 121.354 \\
1,750 & 66.5882 & 147.186 & 152.360 \\
1,875 & 83.2914 & 171.411 & 188.266 \\
\hline
\end{tabular}

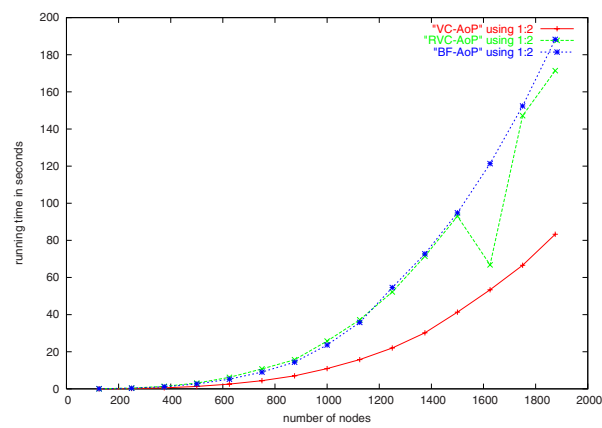

Fig. 10. Comparison of Vertex Contraction (VC), Random Vertex Contraction (RVC) and Bellman-Ford (BF) Array of Pointer (AoP) implementation execution times (seconds) required to solve the Negative Cost Cycle problem for Type E graphs.

\begin{tabular}{|r|c|c|c|}
\hline \multirow{2}{*}{$n$} & \multicolumn{4}{|c|}{$\begin{array}{c}\text { Simple Pointer } \\
\text { (Time in Seconds) }\end{array}$} \\
\cline { 2 - 4 } & VC & RVC & BF \\
\hline 125 & 0.06657 & 0.01769 & 0.04572 \\
250 & 0.48752 & 0.09771 & 0.33976 \\
375 & 1.61851 & 0.47191 & 1.11752 \\
500 & 4.55655 & 0.53125 & 2.61450 \\
625 & 10.2743 & 2.45196 & 5.06600 \\
750 & 19.7552 & 3.40885 & 8.70514 \\
875 & 33.6204 & 5.76912 & 13.7683 \\
1,000 & 52.5211 & 7.97386 & 20.4959 \\
1,125 & 76.4936 & 25.6282 & 29.0852 \\
1,250 & 106.248 & 33.1245 & 39.9211 \\
1,375 & 144.869 & 9.84373 & 53.0680 \\
1,500 & 187.058 & 67.5207 & 69.8548 \\
1,625 & 244.296 & 66.2070 & 91.4537 \\
1,750 & 304.338 & 78.6134 & 118.606 \\
1,875 & 379.341 & 29.4672 & 151.796 \\
\hline
\end{tabular}

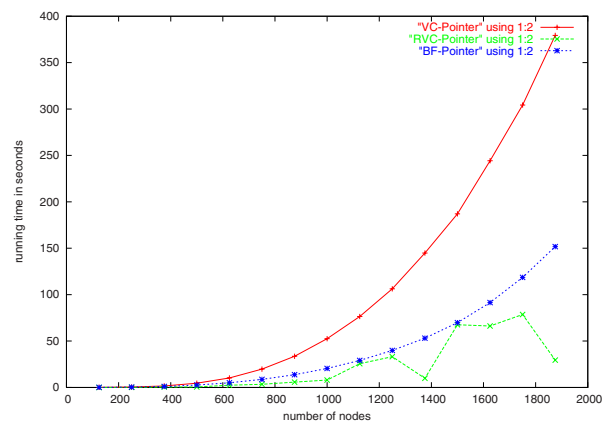

Fig. 11. Comparison of Vertex Contraction (VC), Random Vertex Contraction (RVC) and Bellman-Ford (BF) Simple Pointer implementation execution times (seconds) required to solve the Negative Cost Cycle problem for Type E graphs. 


\subsection{Conclusions}

$\mathrm{VC}$ does considerably better than both RVC and BF, as observed from the table and graph in Figure (10) of the Array of Pointer implementation on Type E graphs. The results of RVC and BF are similar with RVC doing better in most instances.

VC does very poorly, as observed from the table and graph in Figure (11) of the Pointer implementation on Type E graphs. RVC does much better than $\mathrm{VC}$ and outperforms BF by a large margin on most instances. One conclusion that can be drawn from the data is that the time taken by RVC varies greatly depending on the random sequence of vertices chosen.

\section{Conclusion}

In this paper, we designed and analyzed a greedy algorithm called the vertex contraction algorithm (VC) for the negative cost cycle detection problem. Although vertex contraction is asymptotically inferior to the Bellman-Ford algorithm on sparse graphs, it is vastly superior from an empirical perspective.

We are currently working on two extensions: (a) Comparing our strategy with the Goldberg approach, (b) Combining the main idea of our approach, with heuristics such as contracting the vertex with the smallest degree-product.

\section{References}

[CG96] Boris V. Cherkassky and Andrew V. Goldberg. Negative-cycle detection algorithms. In Josep Díaz and Maria Serna, editors, Algorithms-ESA '96, Fourth Annual European Symposium, volume 1136 of Lecture Notes in Computer Science, pages 349-363, Barcelona, Spain, 25-27 September 1996. Springer.

[CLR92] T. H. Cormen, C. E. Leiserson, and R. L. Rivest. Introduction to Algorithms. MIT Press and McGraw-Hill Book Company, 6th edition, 1992.

[DMP91] R. Dechter, I. Meiri, and J. Pearl. Temporal constraint networks. Artificial Intelligence, 49:61-95, 1991.

[Gol95] Andrew V. Goldberg. Scaling algorithms for the shortest paths problem. SIAM Journal on Computing, 24(3):494-504, June 1995.

[Pug92] W. Pugh. The omega test: A fast and practical integer programming algorithm for dependence analysis. Comm. of the ACM, 35(8):102-114, August 1992.

[Sub02] K. Subramani. An analysis of zero-clairvoyant scheduling. In Joost-Pieter Katoen and Perdita Stevens, editors, Proceedings of the $8^{\text {th }}$ International Conference on Tools and Algorithms for the construction of Systems (TACAS), volume 2280 of Lecture Notes in Computer Science, pages 98-112. SpringerVerlag, April 2002.

[WE94] Neil H. Weste and Kamran Eshragian. Principles of CMOS VLSI Design. Addison Wesley, 1994. 